\title{
Nitric Oxide Activity in the Human Coronary Circulation Impact of Risk Factors for Coronary Atherosclerosis
}

\author{
Arshed A. Quyyumi, “ Nader Dakak, ${ }^{\star}$ Neil P. Andrews, ${ }^{\star}$ Syed Husain, ${ }^{\star}$ Sanjeev Arora, ${ }^{\ddagger}$ David M. Gilligan, ${ }^{5}$ Julio A. Panza, ${ }^{\star}$ \\ and Richard O. Cannon III* \\ With the technical assistance of Gregory Johnson and Rita Mincemoyer \\ ${ }^{*}$ Cardiology Branch, National Heart, Lung, and Blood Institute/National Institutes of Health, Bethesda, Maryland 20892; ${ }^{\ddagger}$ University of \\ West Virginia Hospital, Morgantown, West Virginia 26506; and ${ }^{\S}$ Division of Cardiology, Medical College of Virginia, \\ Richmond, Virginia 23298
}

\begin{abstract}
The bioavailability of nitric oxide (NO) in the human coronary circulation at rest and after acetylcholine (ACH)-induced vasodilation was investigated in 32 patients with angiographically normal coronary arteries. The effects of intracoronary $L-N^{G}$ monomethyl arginine (L-NMMA) were investigated at rest and after $\mathrm{ACH}$, sodium nitroprusside, and adenosine. L-NMMA ( $64 \mu \mathrm{mol} / \mathrm{min})$ increased resting coronary vascular resistance by $22 \%(P<0.001)$, reduced distal epicardial coronary artery diameter by $12.6 \%$ ( $P$ $<0.001$ ), and inhibited ACH-induced coronary epicardial and microvascular vasodilation. These effects were reversed with intracoronary L-arginine. L-NMMA did not inhibit dilation in response to sodium nitroprusside and adenosine.

23 patients were exposed to one or more coronary risk factors. The vasoconstrictor effect of L-NMMA on the epicardial and microvessels was greater in patients free of risk factors: Coronary vascular resistance was $36 \%$ higher in patients without risks, compared to $17 \%$ higher in patients with risks $(P<0.05)$. Both epicardial and microvascular dilator effects of $\mathrm{ACH}$ were greater in patients without risk factors, and the inhibition of these effects by L-NMMA was also greater in patients without risk factors.

Thus: (a) NO contributes importantly to resting epicardial and coronary microvascular tone, $(b)$ coronary vascular dilation in response to $\mathrm{ACH}$ is predominantly due to increased production of $\mathrm{NO}$, and (c) despite the absence of angiographic evidence of atherosclerosis, exposure to coronary risk factors is associated with reduced resting and stimulated bioavailability of NO from the human coronary circulation. (J. Clin. Invest. 1995. 95:1747-1755.) Key words: nitric oxide $\cdot$ coronary circulation $\cdot$ endothelium-dependent vasodilation $\cdot$ coronary risk factors
\end{abstract}

\section{Introduction}

The vascular endothelium plays an important role in maintaining blood vessel tone by releasing different dilator and constrictor substances (1-4). Endothelium-derived relaxing factors

Address correspondence to Arshed A. Quyyumi, National Institutes of Health, Cardiology Branch, National Heart, Lung, and Blood Institute, Building 10, Room 7B-15, Bethesda, MD 20892.

Received for publication 15 July 1994 and in revised form 29 September 1994.

The Journal of Clinical Investigation, Inc.

Volume 95, April 1995, 1747-1755 are produced in response to a variety of receptor-dependent and -independent pharmacologic probes, including acetylcholine $(\mathrm{ACH}),{ }^{1}$ substance $\mathrm{P}$, bradykinin, $\mathrm{ADP}$, and calcium ionophore, and also in response to physiologic phenomena such as increases in shear stress (1-9). One important endotheliumderived relaxing factor has been characterized as nitric oxide (NO), or a compound closely related to NO (10-12). The capacity of blood vessels to release NO at rest or with stimulation is variable between organs and species (13-22). Studies of human coronary endothelial function have largely relied on stimulating production of endothelium-derived relaxing factor with $\mathrm{ACH}(8,9,23-31)$. In these investigations, abnormal endothelial function is considered to be present when epicardial coronary arteries constrict and blood flow increase is attenuated with intracoronary $\mathrm{ACH}$, but whether this is an accurate reflection of the state of the endothelium in general, and of NO bioactivity in particular, is unknown. There are sparse data on the basal activity of NO in human coronary vasculature in vivo, and doubts have been raised in a recent study as to whether $\mathrm{ACH}$ causes release of NO from the human coronary vasculature (32). Moreover, although the effect of $\mathrm{ACH}$ is depressed in the presence of risk factors for coronary atherosclerosis (33, 34 ), it is not known whether risk factors adversely affect basal or stimulated production of NO from the human coronary circulation in vivo.

We therefore designed this study to: $(a)$ investigate the role of NO in modulating resting coronary vascular tone, $(b)$ study whether ACH releases NO from the human coronary vasculature, and $(c)$ elucidate the effects of exposure to risk factors for coronary atherosclerosis on NO production from the human coronary arteries.

\section{Methods}

Patients. We studied 32 patients with angiographically normal coronary arteries who were undergoing diagnostic cardiac catheterization for investigation of chest pain or abnormal noninvasive tests. Patients with previous myocardial infarction or valvular heart disease were excluded. There were $15(47 \%)$ men and 17 women with a mean age of $46 \pm 11$ yr. 10 patients were hypertensive (BP $>140 / 90$ ), but none had echocardiographic evidence of left-ventricular hypertrophy. Hypercholesterolemia (total cholesterol $>260 \mathrm{mg} / \mathrm{dl}$ ) was present in 7 patients, 10 were current smokers, 6 had diabetes, and 5 patients were older than $60 \mathrm{yrs}$ of age. 23 patients were exposed to one or more of the aforementioned risk factors, and 9 had none. All cardiac medications were with-

1. Abbreviations used in this paper: $\mathrm{ACH}$, acetylcholine; $\mathrm{CVR}$, coronary vascular resistance; L-NMMA, $\mathrm{L}-\mathrm{N}^{\mathrm{G}}$ monomethyl arginine; NO, nitric oxide. 


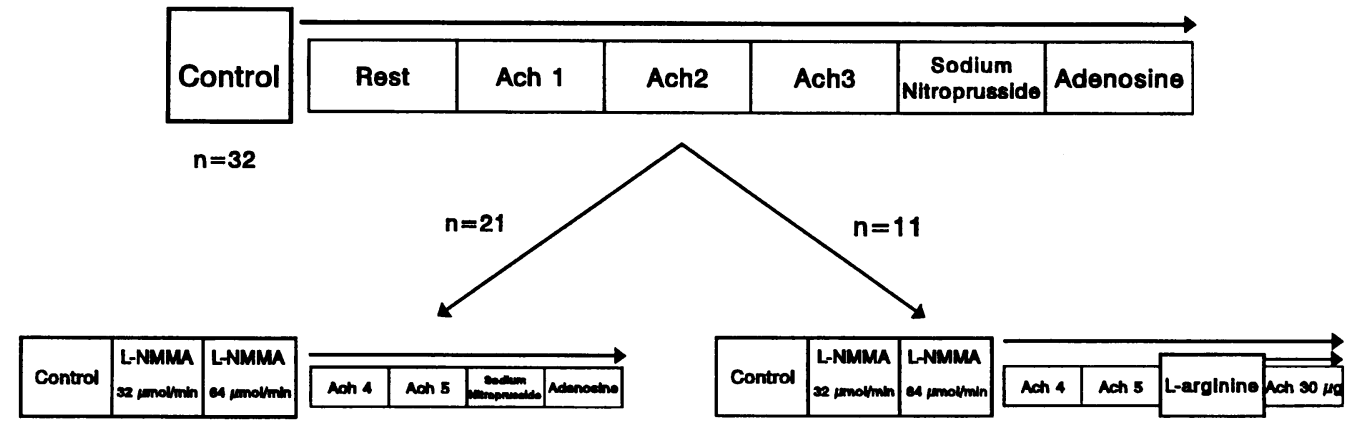

Figure 1. Protocol design. ACH 1, 2, and 3 denote the dose-response curve with ACH. ACH 4 and 5 denote the two highest vasodilating doses of ACH that were reinfused after L-NMMA.

drawn at least $48 \mathrm{~h}$ before the study. Aspirin was discontinued for at least $7 \mathrm{~d}$, and cholesterol-lowering medications were stopped 1 mo before the study in the one patient on this therapy. The study was approved by the National Heart, Lung, and Blood Investigational Review Board, and informed written consent was obtained from all patients.

Protocol. After completion of diagnostic coronary arteriography, a 6-French guide catheter was introduced into the left main coronary artery, and blood flow velocity was measured using a 0.018 -inch wire equipped with a Doppler crystal at its tip (Cardiometrics Flowire; Cardiometrics, Inc., Sunnyvale, CA) $(35,36)$. The Doppler flow wire was advanced into either the left main $(n=6)$ or the proximal segment of a major epicardial coronary artery (left anterior descending coronary artery in 22 patients and the circumflex coronary artery in 4 patients). The wire tip was carefully positioned in a segment of the vessel that was straight, was free of any branches $1 \mathrm{~cm}$ from the tip, produced an adequate flow velocity signal, and could be imaged without overlap from other vessels, thus allowing for quantitative measurements of the coronary artery diameter. All drugs were infused directly into the left main coronary artery via the guide catheter at infusion rates ranging from 1 to $2 \mathrm{ml} / \mathrm{min}$. After a 5 -min infusion of dextrose $5 \%$ at $1 \mathrm{ml} /$ min, baseline coronary blood flow velocity and coronary angiography were performed. A 7-French multipurpose A2 catheter was inserted via the right internal jugular vein into the midcoronary sinus for blood sampling. Oxygen saturation of arterial and coronary sinus venous blood was measured using an oximeter in 23 patients at baseline and after infusions of $\mathrm{L}-\mathrm{N}^{\mathrm{G}}$ monomethyl arginine (L-NMMA; Calbiochem-Novabiochem Corp., La Jolla, CA).

Endothelium-dependent vasodilation was estimated by performing a dose-response curve with incremental infusions of intracoronary $\mathrm{ACH}$ starting at $3 \mu \mathrm{g} / \mathrm{min}$ (Fig. 1). After $2 \mathrm{~min}$, blood flow velocity and coronary angiography were performed. This was followed by 2-min infusions of 30,100 , and $300 \mu \mathrm{g} / \mathrm{min}$ of intracoronary ACH with measurement of Doppler flow velocity and angiography after each increment. ACH dose was not increased further once an infusion either reduced blood flow velocity or severely $(>50 \%)$ narrowed the epicardial coronary tree. Thus, all patients received the $30-\mu \mathrm{g} / \mathrm{min}$ dose, 18 received doses up to $100 \mu \mathrm{g} / \mathrm{min}$, and 13 received doses up to $300 \mu \mathrm{g} /$ $\mathrm{min}$. The peak flow response with $\mathrm{ACH}$ was achieved at the $30-\mu \mathrm{g} / \mathrm{min}$ dose in 18 patients, at the $100-\mu \mathrm{g} / \mathrm{min}$ dose in 12 patients, and at the $300-\mu \mathrm{g} / \mathrm{min}$ dose in 2 patients.

5 min after performing the $\mathrm{ACH}$ dose-response curve, endotheliumindependent function was estimated with sodium nitroprusside and adenosine (Fig. 1). Intracoronary sodium nitroprusside was given at $40 \mu \mathrm{g} /$ min for $3 \mathrm{~min}$, followed by measurement of blood flow velocity and coronary angiography. This was followed by administration of intracoronary adenosine at $2.2 \mathrm{mg} / \mathrm{min}$ for $2 \mathrm{~min}$. Peak flow velocity measurement and angiography were repeated.

After a 10-min interval, while continuing dextrose 5\% infusion, repeat baseline measurement of flow velocity, oxygen saturations, and angiography were made (Fig. 1). These were followed by infusion of
L-NMMA, a specific inhibitor of NO synthesis from L-arginine (37). L-NMMA was infused at $32 \mu \mathrm{mol} / \mathrm{min}(0.5 \mathrm{ml} / \mathrm{min})$ for $5 \mathrm{~min}$ and then increased to $64 \mu \mathrm{mol} / \mathrm{min}(1 \mathrm{ml} / \mathrm{min})$ for another $5 \mathrm{~min}$. Blood flow velocity, coronary angiography, and arteriovenous oxygen saturations were measured at each stage.

While continuing the infusion of L-NMMA at $64 \mu \mathrm{mol} / \mathrm{min}, \mathrm{ACH}$ was readministered in 30 patients at the two vasodilating doses that produced the highest increase in flow velocity. 21 patients had repeat infusion of $40 \mu \mathrm{g} / \mathrm{min}$ sodium nitroprusside for $3 \mathrm{~min}$, and adenosine $2.2 \mathrm{mg} / \mathrm{min}$ was reinfused in 15 patients (Fig. 1). Blood flow velocity was measured and coronary angiography was performed after each intervention. In the remaining 11 patients, $\mathrm{L}$-arginine was administered to test whether the effects of L-NMMA were reversible (Fig. 1). $10 \mathrm{~min}$ after intracoronary L-arginine $(160 \mu \mathrm{mol} / \mathrm{min}$ at $1 \mathrm{ml} / \mathrm{min})$, repeat measurements of blood flow velocity and coronary angiography were performed. ACH was reinfused at $30 \mu \mathrm{g} / \mathrm{min}$ for $2 \mathrm{~min}$ in 10 patients.

Estimation of coronary blood flow and diameter. Coronary blood flow was estimated from measurement of coronary blood flow velocity and diameter measurements using the formula ( $\pi \times$ average peak velocity $\times 0.125 \times$ diameter $\left.^{2}\right)$. Coronary vascular resistance $(C V R)$ was calculated as mean arterial pressure divided by coronary blood flow.

For calculating flow, coronary artery diameter was measured in a $0.5-\mathrm{cm}$ segment of vessel beginning $0.25 \mathrm{~cm}$ beyond the tip of the flow wire. Coronary angiograms were recorded using a cineangiographic system (Toshiba American Medical Systems Inc., Malvern, PA). Quantitative angiography was performed with the ARTEK software (Quantim 200I, StatVIEW; ImageComm Systems, Inc., Sunnyvale, CA). In addition to measurement of the diameter at the level of the Doppler flow wire, $0.5-1 \mathrm{~cm}$ segments of the proximal and distal segments of the epicardial coronary arteries were also measured by quantitative coronary angiography.

Statistical analysis. Data are expressed as mean \pm SD in the text and mean $\pm S E$ in figures. Differences between means were compared by paired or unpaired Student's $t$ test, as appropriate. The effect of LNMMA on the two doses of ACH, and the effects of two doses of LNMMA on patients with and without risk factors, were compared by ANOVA for repeated measures using a multiple regression model that included dummy variables to correct for between-subject variability (38). The differences between the effects of L-NMMA in patients with and without risk factors were compared using the percent change from baseline for all parameters because of the baseline differences in regional diameters and flow in the two subgroups. All $P$ values are two tailed, and a value $<0.05$ is considered of statistical significance. Where the effect of two doses of L-NMMA on coronary hemodynamics is compared with baseline, a paired Student's $t$ test was performed using a Bonferonni adjustment to alpha.

\section{Results}

Coronary vascular response to inhibition of $N O$ synthesis with L-NMMA. Epicardial coronary arteries progressively constricted 


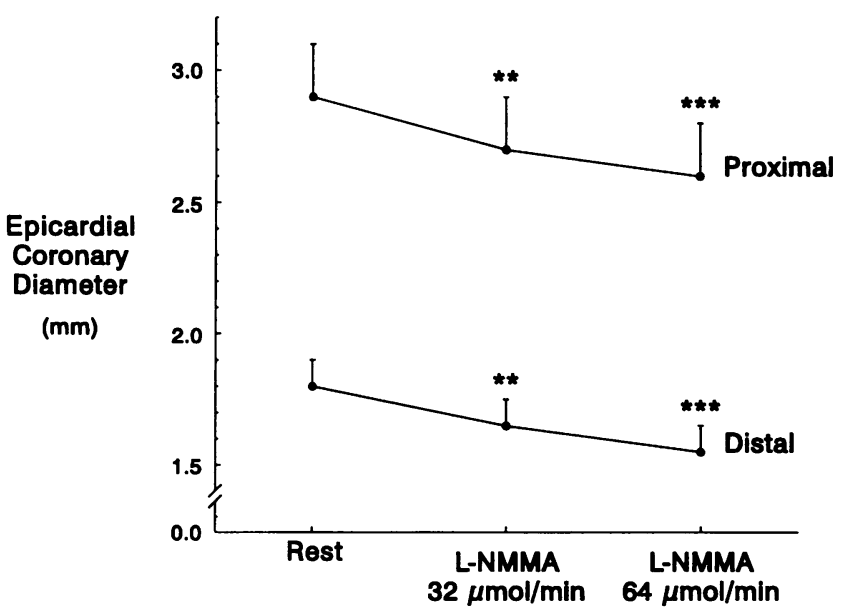

Figure 2. Proximal and distal epicardial coronary artery diameter changes with L-NMMA. Data represent mean \pm SEM. ${ }^{* *} P<0.01$, $* * * P<0.001$ compared to rest.

with increasing doses of L-NMMA (Fig. 2). Proximal and distal segments of the epicardial coronary arteries constricted by 5.3 and $7.4 \%$, respectively, with the lower dose of L-NMMA, and by 9.2 and $12.6 \%$, respectively, at the $64 \mu \mathrm{mol} / \mathrm{min}$ dose, demonstrating tonic basal release of NO from the coronary epicardial vessels. The differences between proximal and distal segments were not significant.

CVR also progressively increased with L-NMMA (22\% with the $64 \mu \mathrm{mol} / \mathrm{min}$ dose), indicating tonic release of NO from the coronary microvessels under resting conditions (Fig. 3 ). Arterial pressure increased by a mean of $2.9 \%$ at the lower dose and $6.8 \%$ at the higher dose of L-NMMA. Arterio-venous

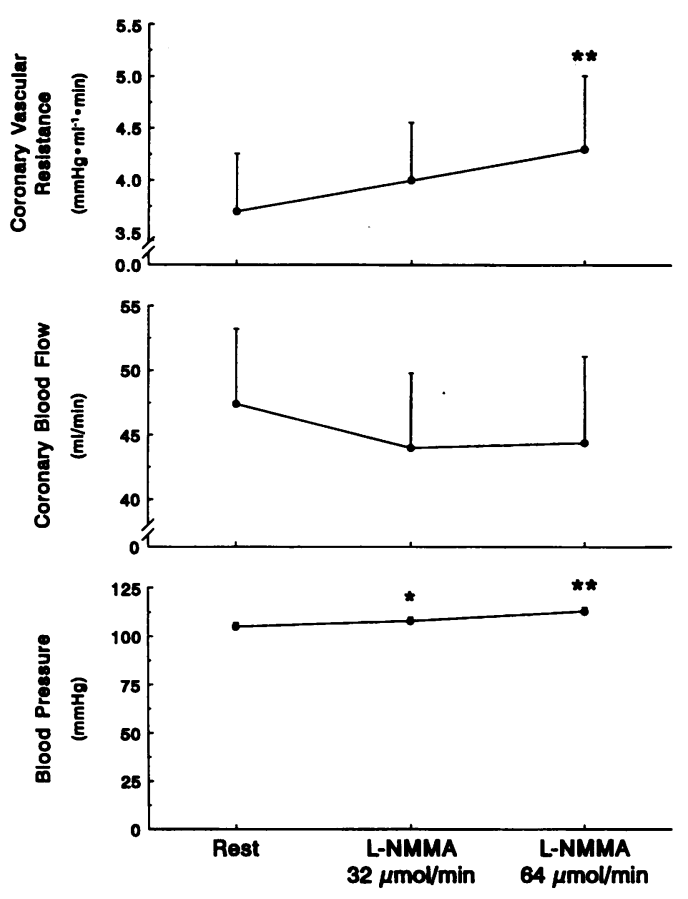

Figure 3. Changes in coronary vascular resistance, blood flow, and mean arterial pressure with increasing concentrations of L-NMMA. Data represent mean \pm SEM. ${ }^{*} P<0.025, * * P<0.01$ compared to rest.
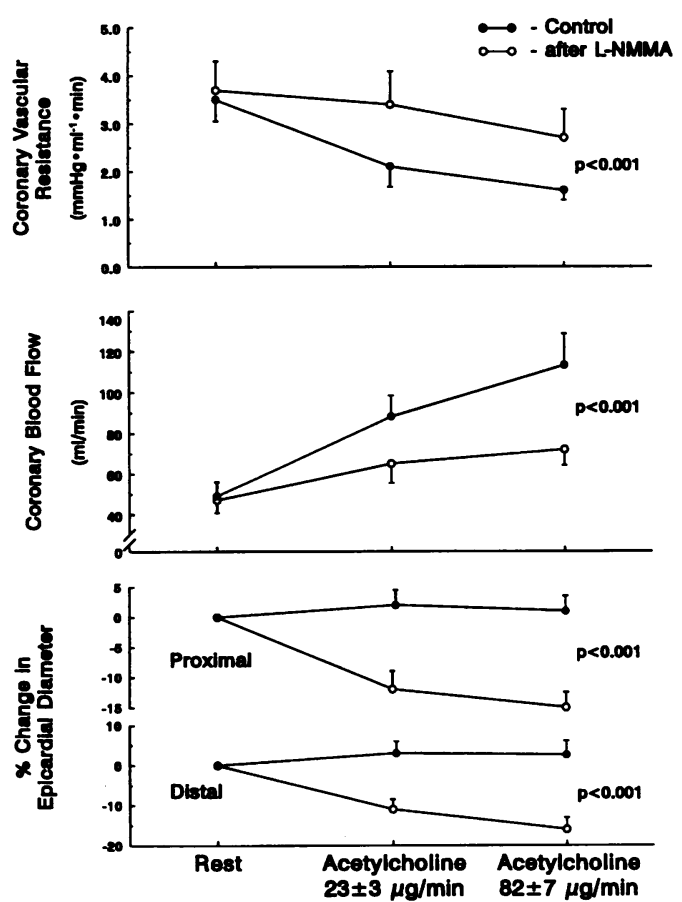

Figure 4. Effects of increasing doses of intracoronary $\mathrm{ACH}$ on coronary vascular resistance, flow, and the percent change in proximal and distal epicardial coronary artery diameters before (solid circles) and after (open circles) L-NMMA. Two vasodilating doses of ACH were reinfused after L-NMMA, and the $P$ values denote results of ANOVA comparing the response to ACH before vs after L-NMMA. Data represent mean \pm SEM.

oxygen difference widened from $56 \pm 7$ to $59 \pm 6 \%(P=0.003)$ with the higher dose of L-NMMA, while the rate-pressure product remained unchanged $\left(8,736\right.$ before to $8,958 \mathrm{mmHg} \cdot \mathrm{bpm}^{-1}$ after L-NMMA).

Effect of L-NMMA on the response to $A C H$. ACH infusions produced graded increases in coronary blood flow and reduction in coronary vascular resistance. At the peak vasodilating dose of $\mathrm{ACH}$, coronary blood flow increased by $161 \%$ and CVR decreased by $52 \%$. Coronary artery diameter changes were heterogenous, but in the group as a whole there was no significant alteration in either the proximal or the distal coronary artery diameters.

When ACH was reinfused at the two highest vasodilating doses, there was significant inhibition of $\mathrm{ACH}$-induced epicardial and microvascular dilation (Fig. 4). Thus, at the lower concentration of $\mathrm{ACH}$, flow was $26 \%$ lower and CVR $61 \%$ higher, and, at the higher concentration, flow was $28 \%$ lower and CVR 85\% higher after L-NMMA $(P<0.003)$.

An insignificant change in epicardial coronary artery diameter with $\mathrm{ACH}$ alone was converted to a net reduction after LNMMA in the proximal and distal segments (Fig. 4). Because the coronary artery diameters during the initial baseline study tended to be lower compared to the pre-L-NMMA baseline ( $P$ $=\mathrm{NS}$ ), diameter changes with $\mathrm{ACH}$ were compared as percent change from baseline before and after L-NMMA. Thus, at the higher dose of $\mathrm{ACH}$, proximal and distal segments of epicardial coronary arteries dilated by 1.2 and $2.6 \%$, respectively. After L-NMMA, the same dose of ACH produced 15 and $16 \%$ ( $P$ $<0.001$ ) reduction in diameter, respectively. 

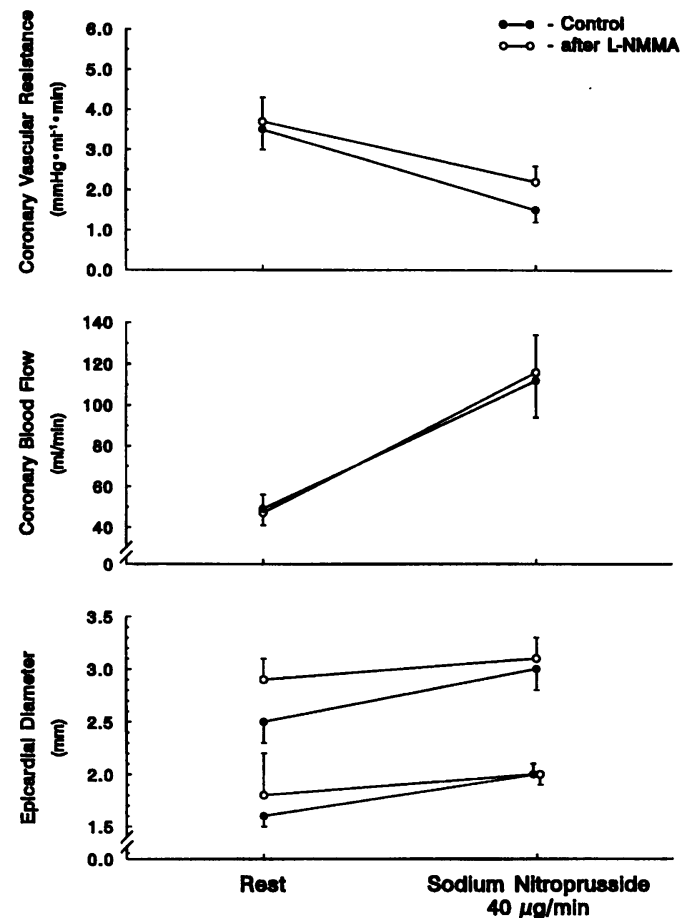

Figure 5. Effect of L-NMMA on the coronary vascular resistance, flow, and proximal and distal epicardial coronary artery diameters with intracoronary sodium nitroprusside. Control study is indicated by solid circles, and post-L-NMMA study by open circles. Differences were not statistically significant. Data represent mean \pm SEM.

Effect of L-NMMA on the response to sodium nitroprusside. Coronary blood flow increased by $137 \%$, CVR was reduced by $56 \%$, and blood pressure fell by a mean of $10 \mathrm{mmHg}$ with 40 $\mu \mathrm{g} / \mathrm{min}$ infusion of sodium nitroprusside during the control study. After L-NMMA, the increase in blood flow and reduction in CVR with sodium nitroprusside were similar; 137 and $-56 \%$ change, respectively, $P=$ NS (Fig. 5). Thus, CVR with sodium nitroprusside was similar before and after L-NMMA. Similarly, epicardial coronary artery diameters were similar in the proximal and distal segments with sodium nitroprusside before and after L-NMMA (Fig. 5).

Effect of L-NMMA on the response to adenosine. Coronary blood flow increased by $396 \%$ and CVR decreased by $76 \%$ during the control study, and by 376 and $72 \%$, respectively, after L-NMMA ( $P=$ NS) (Fig. 6). Coronary diameter increased to similar levels with adenosine before and after L-NMMA in the proximal and distal segments (Fig. 6).

Effect of L-arginine on the response to L-NMMA at rest. The results of concomitant administration of L-arginine and LNMMA on resting coronary vascular hemodynamics are illustrated in Fig. 7. The L-NMMA-induced increase in resting CVR and reduction in distal coronary artery diameter were reversed by L-arginine.

Effect of L-arginine on the response to L-NMMA and ACH. $\mathrm{ACH}$ at $30 \mu \mathrm{g} / \mathrm{min}$ produced a $47 \%$ reduction in the coronary vascular resistance before, but only a $9 \%$ reduction after LNMMA (Fig. 8). After L-arginine, this inhibitory effect of LNMMA on endothelium-dependent vasodilation with $\mathrm{ACH}$ was reversed (CVR reduced by 48\%) (Fig. 8). Similarly, epicardial coronary arteries, particularly in the distal coronary segments,
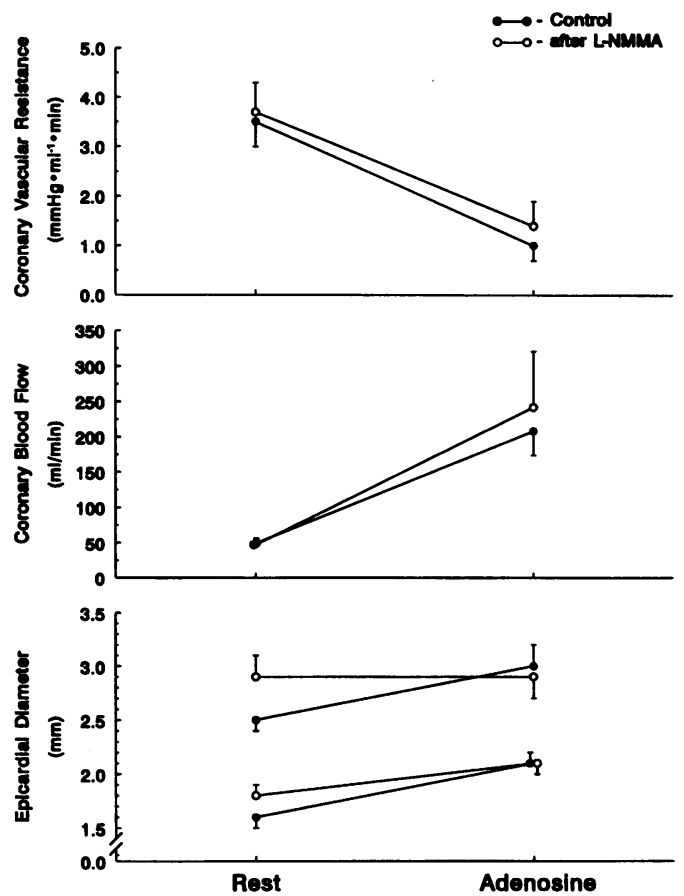

Figure 6. Effect of L-NMMA on the coronary vascular resistance, flow, and proximal and distal epicardial coronary artery diameters with intracoronary adenosine. Control study is indicated by solid circles, postL-NMMA study by open circles. Differences were not statistically significant. Data represent mean \pm SEM.

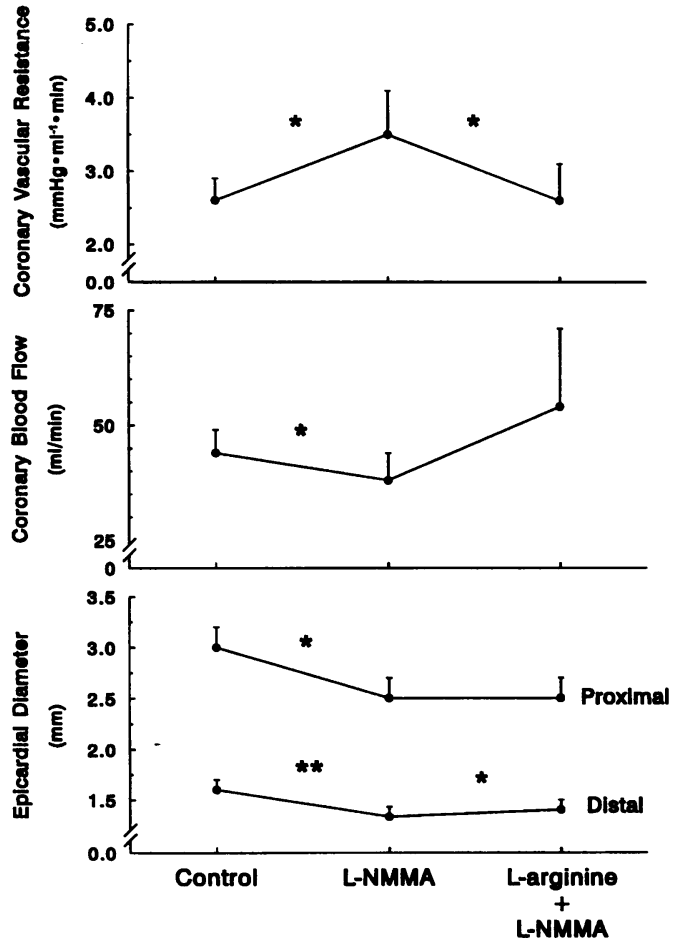

Figure 7. The effect of L-arginine on the coronary vascular effects of L-NMMA at rest. Coronary vascular resistance, flow, and proximal and distal epicardial coronary diameters are compared at rest (control), after L-NMMA, and after L-NMMA and L-arginine. Results are expressed as mean \pm SEM. ${ }^{*} P<0.05,{ }^{* *} P<0.01$ 


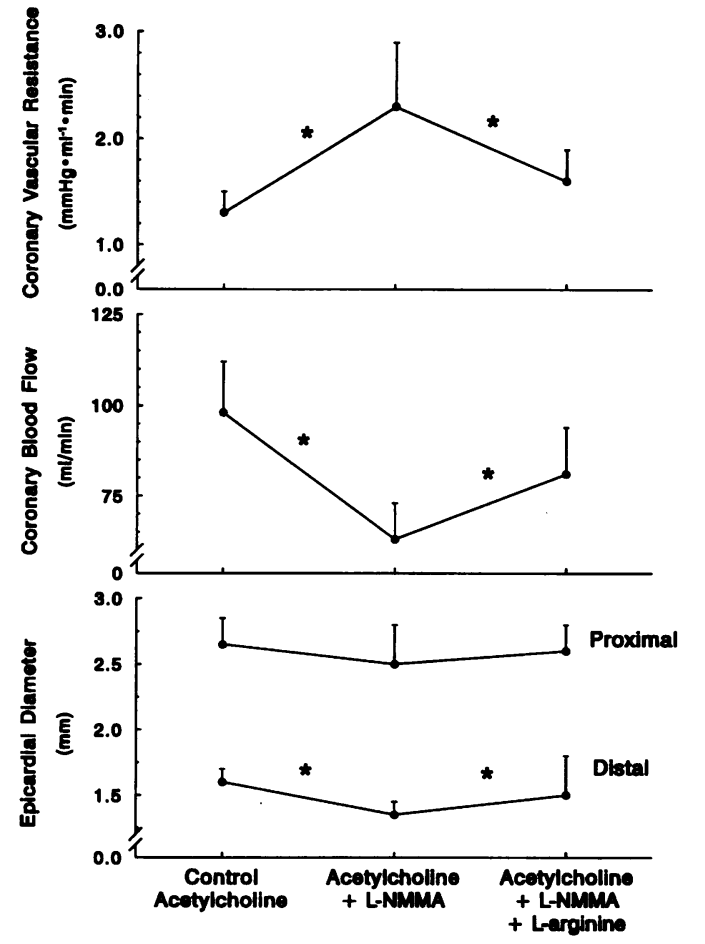

Figure 8. The effect of L-arginine on the response to $\mathrm{ACH}$ before and after L-NMMA. Coronary vascular resistance, flow, and proximal and distal epicardial coronary diameters with $\mathrm{ACH}$ are compared under resting conditions (control), after L-NMMA, and after L-arginine and L-NMMA. Results are expressed as mean \pm SEM. ${ }^{*} P<0.05$.

were more constricted when ACH was administered with LNMMA compared with the control study. L-arginine counteracted this constrictive effect of L-NMMA.

The impact of coronary risk factors on the response to $L$ NMMA at rest. The responses of epicardial coronary arteries and of the coronary microvasculature to L-NMMA under resting conditions were heterogenous, ranging from a decrease in CVR of $29 \%$ to an increase of $99 \%$ at the higher dose of L-NMMA, suggesting variability in the basal activity of NO. To investigate whether this heterogeneity was related to the presence of risk factors for coronary atherosclerosis, the responses in patients without risk factors were compared to the responses in patients with risk factors for coronary atherosclerosis. Risk factors for coronary atherosclerosis were.defined as serum cholesterol $>260 \mathrm{mg} / \mathrm{dl}$, hypertension $>140 / 90 \mathrm{mmHg}$, age $>60 \mathrm{yr}$, history of current smoking, or diabetes. 9 patients were exposed to none of these risk factors, whereas the remaining 23 had exposure to one or more risk factors.

Coronary microvascular and epicardial vessel changes in response to L-NMMA at rest in patients with and without risk factors for coronary atherosclerosis are shown in Fig. 9. The inhibitory effect of L-NMMA on the coronary microvasculature was greater in patients without risk factors. The mean $36 \%$ increase in CVR and $18 \%$ reduction in coronary blood flow (both $P<0.01$ compared to baseline) with the higher dose of $L$ NMMA in patients without risk factors was significantly greater compared to patients with risk factors, who had a $17 \%$ increase in CVR ( $P=0.08$ compared to baseline $)$ and $3 \%$ fall ( $P=$ NS compared to baseline) in blood flow. Although significant epicardial vessel constriction occurred with L-NMMA in both

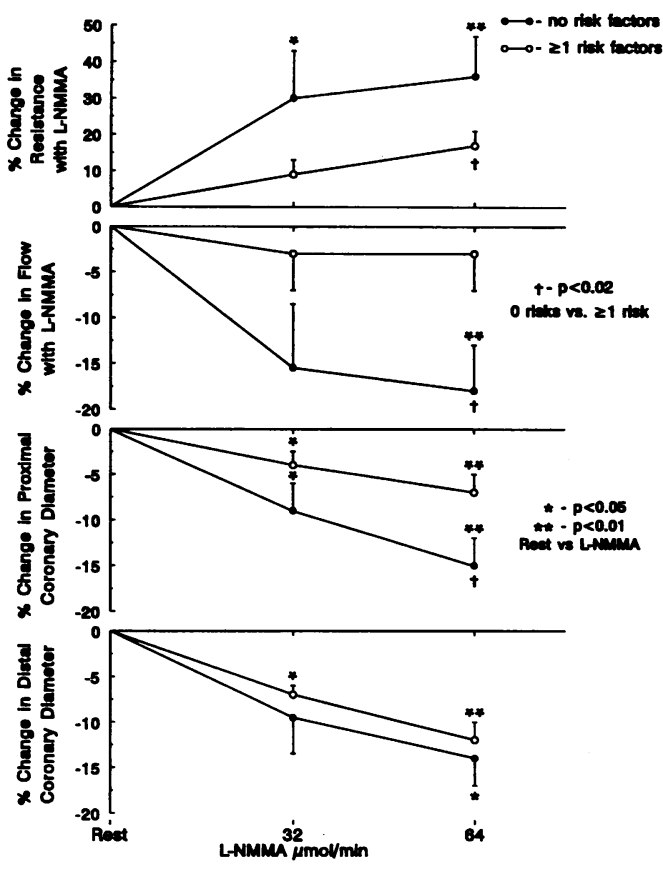

Figure 9. The effect of risk factors for coronary atherosclerosis on the change in resting coronary vascular tone with L-NMMA. The percent change in the coronary vascular resistance, flow, and proximal and distal epicardial diameters with L-NMMA are compared in patients with $\geq 1$ risk factor (open circles) and in patients without risk factors (solid circles). Results are expressed as mean $\pm \mathrm{SEM} .{ }^{\dagger} P<0.001$, and denotes differences in the response to L-NMMA by ANOVA between patients with and without risk factors. $* P<0.05$, and $* * P<0.01$ denote differences between rest and post-L-NMMA measurements in each group.

groups, the magnitude of constriction appeared to be greater in patients without risk factors and reached statistical significance in the proximal coronary artery segments $(P<0.02)$, but not in the distal segments $(P=0.07)$ (Fig. 9).

Effect of L-NMMA on the response to ACH in patients with risk factors. The peak microvascular dilator response with $\mathrm{ACH}$ (69\% fall in CVR) was significantly higher in patients without risk factors than in those with one or more risk factors $(47 \%$ fall in CVR) (Fig. 10). At this concentration of $\mathrm{ACH}$, epicardial coronary arteries dilated in patients without risk factors, whereas there was constriction in patients with risk factors. To investigate whether these differences represented differences in stimulated release of NO in these patients, the effect of L-NMMA was studied.

L-NMMA inhibited the microvascular dilation in response to ACH in both groups of patients, but the magnitude of inhibition was significantly greater in patients without risk factors: CVR increased by $116 \%$ in patients without risk factors compared with a $70 \%$ increase in those with risk factors $(P<0.02)$. Similarly, distal epicardial diameter decreased by $18 \%$ in patients without risks compared with a 5\% decrease in those with risk factors $(P<0.01)$. Thus, after L-NMMA, the percent increase in flow, the reduction in resistance, and the reduction in epicardial diameter with $\mathrm{ACH}$ were similar in both groups (Fig. 10).

Effect of L-NMMA on the responses to sodium nitroprusside and adenosine in patients with risk factors. Baseline microvas- 


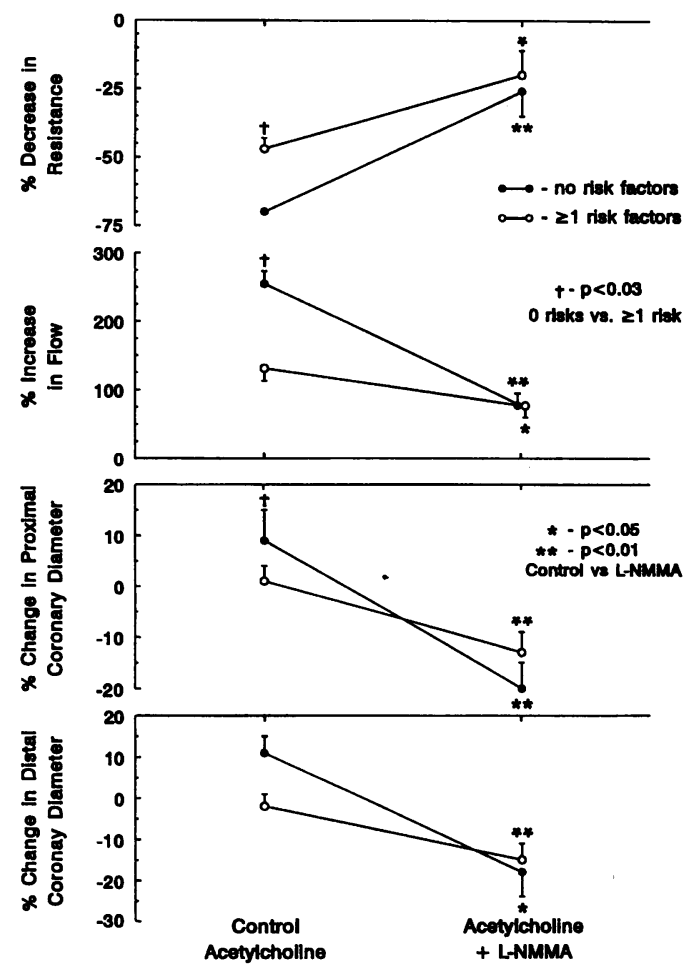

Figure 10. The impact of risk factors for coronary atherosclerosis on the vascular effects of ACH before and after L-NMMA. The percent change in coronary vascular resistance, flow, and proximal and distal epicardial coronary diameters with $\mathrm{ACH}$ in patients with $\geq 1$ risk factor (open circles) is compared to that in patients without risk factors (solid circles). Responses to the peak dose of $\mathrm{ACH}$ are compared before and after L-NMMA in the two groups. Results are expressed as mean \pm SEM. ${ }^{\dagger} P<0.03$, and denotes differences in the response to ACH between patients with and without risk factors under control conditions. ${ }^{*} P$ $<0.05$ and $* * P<0.01$ denote differences between control and postL-NMMA measurements in each group.

cular vasodilation with sodium nitroprusside was similar in patients with and without risk factors: a $58 \pm 20 \%$ reduction in CVR in patients without vs a $59 \pm 16 \%$ decrease in patients with risk factors. Coronary diameter increases were also similar: $23 \pm 10$ vs $27 \pm 19 \%$ increase in distal epicardial diameter in patients without and with risk factors, respectively. After LNMMA, epicardial diameter and CVR with sodium nitroprusside remained unchanged in both groups $(P=\mathrm{NS})$.

Vasodilation in response to adenosine was also similar in both groups: CVR $80 \pm 2 \%$ lower in patients without compared with $77 \pm 8 \%$ in patients with risk factors $(P=\mathrm{NS})$. Coronary diameter increases with adenosine were also similar: $28 \pm 5$ vs $29 \pm 19 \%$ increase in distal epicardial diameter in patients without and with risk factors, respectively. After L-NMMA, epicardial diameter and CVR with adenosine remained unchanged in both groups $(P=\mathrm{NS})$.

\section{Discussion}

\section{NO and basal epicardial coronary artery tone}

There was progressive constriction of epicardial coronary arteries with increasing concentrations of intracoronary L-NMMA. In patients with normal coronary arteries who were not exposed to risk factors for atherosclerosis, segments of proximal and distal epicardial coronary arteries constricted by $9-10 \%$ at the lower dose and between 14 and $15 \%$ at the higher dose of $L-$ NMMA (Fig. 9). There did not appear to be any quantitative difference in the effect of L-NMMA in the proximal compared to the distal sections of the normal epicardial coronary tree. These data provide evidence for tonic release of NO from coronary epicardial vessels under resting conditions in humans. Since L-NMMA is a competitive antagonist and therefore may not have completely blocked all NO production, the results indicate that at least $15 \%$ of resting epicardial coronary vasodilator tone is due to NO.

\section{NO and basal coronary microvascular tone}

The progressive reduction in coronary blood flow and increase in CVR with incremental concentrations of L-NMMA demonstrate that there is also tonic release of NO from the coronary microvasculature under resting conditions. Thus, in patients without coronary risk factors for atherosclerosis, coronary blood flow was $18 \%$ lower and CVR 36\% higher after the higher dose of L-NMMA, suggesting that a significant proportion of resting coronary microvascular tone is influenced by tonic release of NO (Fig. 9). That this effect of L-NMMA was specific for inhibiting L-arginine metabolism to NO was suggested by the fact that its effects were reversed by L-arginine at a concentration that does not have intrinsic vasodilator effects (Fig. 7) $(39,40)$.

Since CVR is believed to be determined predominantly by arterioles $<200 \mu \mathrm{m}$ in diameter in a setting of normal epicardial coronary arteries (41), and because CVR is inversely related to the fourth power of the radius of these resistance vessels, it can be estimated from our results that the diameter of these vessels would need to be reduced by $\sim 7.5 \%$ to produce a $36 \%$ increase in CVR. Thus, the magnitude of effect in terms of change in diameter appears to be lower in the resistance vessels compared to the epicardial vessels, suggesting that the contribution of NO to vascular tone in the normal human coronary circulation is dependent on the size of the coronary vessels.

Compared to the increase in microvascular forearm vascular resistance observed in normal individuals, which has been reported to vary between 40 and $50 \%(42-45)$, the $36 \%$ increase in CVR observed in patients without risk factors appears to be similar and indicates equivalent contribution of NO to resting blood vessel tone in the coronary and forearm vascular beds in humans.

\section{$A C H$ releases NO from the human coronary vasculature}

Epicardial coronary arteries. Several investigators have interpreted the effects of $\mathrm{ACH}$ on human coronary arteries as indicators of endothelial function, but the role of $\mathrm{ACH}$ in releasing NO from human coronary vessels remains controversial (32). Our study unequivocally demonstrates that coronary epicardial vasodilation with $\mathrm{ACH}$ is in large part due to release of NO. In patients without risk factors, $\mathrm{ACH}$-induced epicardial vasodilation was abolished and converted to vasoconstriction in all sections of the coronary epicardial tree after L-NMMA, suggesting that $\mathrm{ACH}$-induced dilation of normal coronary arteries is predominantly due to release of NO. Additionally, it appears that, in the absence of endothelium, the direct effect of $\mathrm{ACH}$ on human coronary epicardial vessels is constriction, as indicated by the response of ACH after L-NMMA. Our data also indicate that the epicardial coronary arteries of patients with 
risk factors release $\mathrm{NO}$ with $\mathrm{ACH}$, as evidenced by the significant further constriction that occurred with ACH after LNMMA. The bioavailability of NO, however, is lower in patients with risk factors compared to those free of risk factors.

Coronary microvessels.' Coronary microvascular dilation due to ACH in patients free of risk factors was significantly inhibited by L-NMMA, suggesting that $\mathrm{ACH}$ also promotes release of NO from human coronary microvessels. However, lack of complete inhibition of $\mathrm{ACH}$-induced dilation of the microvessels suggests, but does not confirm, a non-NO component of $\mathrm{ACH}$-induced vasodilation in human coronary vessels. Whether this is due to release of endothelium-derived hyperpolarizing factor (4) needs to be further investigated. An alternative explanation for persistent vasodilation of the coronary microvasculature with ACH after L-NMMA is that there is incomplete inhibition of NO synthase by the dose of L-NMMA employed in this study.

\section{Specificity of inhibition of NO synthesis by L-NMMA}

The effect of L-NMMA on the coronary microvasculature was specific for inhibiting endothelium-dependent effects of $\mathrm{ACH}$ because the responses of coronary microvessels to sodium nitroprusside, a direct donor of NO, and to adenosine, both endothelium-independent dilators, were unaffected by L-NMMA (Figs. 5 and 6). Moreover, the effect of L-NMMA on vascular tone was reversed by L-arginine, suggesting that the effect of the Larginine analogue was specifically due to competitive inhibition of the L-arginine-NO synthase interaction.

The epicardial diameters with adenosine and sodium nitroprusside before and after L-NMMA were similar (Figs. 5 and 6 ), suggesting that the effect of L-NMMA on epicardial vessels is specific for endothelium-dependent vasodilation, a finding supported by the restoration of the baseline constriction with LNMMA by L-arginine in the distal coronary segments (Fig. 7). However, when the diameters are considered as percent change from baseline, the increase was lower after L-NMMA with both vasodilators, a manifestation of the slightly higher baseline epicardial diameter before L-NMMA was given compared to the baseline at the beginning of the study. There are two possible explanations for this finding. One is that both adenosine and sodium nitroprusside cause maximal or near-maximal epicardial dilation before and after L-NMMA, and the lower percent increase after L-NMMA was merely due to a higher baseline diameter before the second study. Alternatively, the lower percent increase in diameter after L-NMMA could be due to inhibition of flow-mediated . vasodilation by L-NMMA (6-9).

The responses of L-NMMA at rest and after $\mathrm{ACH}$ were reversed by a $10-\mathrm{min}$ infusion of $160 \mu \mathrm{mol} / \mathrm{min}$ of $\mathrm{L}$-arginine. However, this reversal was not significant with respect to the proximal coronary diameter (Figs. 7 and 8). This incomplete reversal may have occurred because L-NMMA was administered for at least $20 \mathrm{~min}$, whereas L-arginine was only given for $10 \mathrm{~min}$ before measurements were made. This may not have allowed sufficient time to overcome all the inhibitory effects of L-NMMA.

\section{Comparison with previous studies}

Our results are compatible with some animal studies that have examined the role of NO on basal coronary vascular tone (14, $15,20)$ but differ from other studies $(18,21,40)$. The contribution of NO to coronary epicardial and microvascular tone in humans in this study appears to be substantially higher than that estimated by Lefroy et al. (32). This difference might be explained either by the smaller concentration of L-NMMA employed, or because coronary blood flow was not directly measured in their study.

Whereas some animal experiments using analogues of $\mathrm{L}$ arginine to block NO synthesis have demonstrated reduced vasodilation of epicardial and coronary microvessels with $\mathrm{ACH}$ $(17-19,21,46)$, others have failed to demonstrate inhibition of ACH-induced vasodilation with L-NMMA (20). A study using L-NMMA in human coronary arteries (32) demonstrated inhibition of $\mathrm{ACH}$-induced dilation of distal epicardial coronary arteries only, but no effect was observed in either the proximal epicardial vessels or the coronary microvessels. Our results unequivocally demonstrate that $\mathrm{ACH}$ stimulates release of $\mathrm{NO}$ from the human epicardial and microvascular coronary circulation. In view of the wide diversity in NO bioavailability that has been reported in different species, it was important in this study to quantitate the role of $\mathrm{NO}$ and $\mathrm{ACH}$ in the human coronary circulation.

\section{Effect of risk factors on NO release from the coronary vasculature}

This study demonstrates, for the first time in the human coronary circulation, that the presence of risk factors for atherosclerosis is associated with a reduced basal activity of NO in both the epicardial coronary arteries and the coronary microvessels. This was demonstrated as a reduced vasoconstrictor action of $\mathrm{L}^{-}$ NMMA in patients with risk factors. Our study nevertheless does not elucidate the mechanism for the depressed bioavailability of NO in these patients. This may be due to a lower rate of synthesis of NO, which may in turn result from substrate deficiency $(39,40)$, or it may result from a defect in the signal transduction pathways (47) or in the enzyme itself. Alternatively, the depressed bioavailability of NO may be secondary to increased breakdown of normally produced NO by superoxide anions $(48,49)$ in patients with risk factors. Also, we are unable to determine whether there are differences in the magnitude of reduction of the bioavailibility of NO with the presence of specific risk factors such as hypertension, hypercholesterolemia, diabetes, smoking, or aging, because of the relatively small number of patients with single risk factors enrolled in this study.

The dilator responses to ACH from the epicardial and microvascular circulation were diminished in patients with risk factors, a finding that is similar to other reports $(33,34,50-52)$. We have additionally confirmed, using the L-arginine analogue, that, in the presence of risk factors, not only is the basal effect of NO reduced, but the stimulated release of NO (in response to $\mathrm{ACH}$ ) is also depressed. Because the effect of $\mathrm{ACH}$ after LNMMA is similar in patients with and without risk factors (Fig. 10 ), it appears that the attenuated response to $\mathrm{ACH}$ in patients with risk factors is due specifically to reduced availability of NO, and not due to an abnormality of other non-NO endothelium-derived relaxing factors or constricting factors.

The epicardial and microvascular dilator responses to sodium nitroprusside and adenosine were similar in patients with and without risk factors, indicating that the blunted $\mathrm{ACH}$ response observed in the latter group is indeed an expression of impaired endothelium-dependent relaxation and not a consequence of reduced responsiveness of the vascular smooth muscle, either to NO-donating dilators like sodium nitroprusside or to non-NO-producing vasodilators such as adenosine. These 
findings are in agreement with other reports demonstrating depressed $\mathrm{ACH}$ responses and normal smooth muscle vasodilation in patients with atherosclerosis $(27,31,33,50-52)$.

It is also important to recognize that abnormalities in the activity of NO in the coronary epicardial vasculature were observed in the absence of any clear angiographic evidence of atherosclerosis. Intimal thickening, observed by intravascular ultrasound, may be present in patients with risk factors despite the absence of angiographic irregularities $(53,54)$; this is associated, in vitro, with reduced production of NO (51) and, in vivo, with an abnormal ACH response (53). Moreover, coronary microvessels that do not develop structural changes in atherosclerosis also have reduced bioavailability of NO in the presence of risk factors. These findings are compatible with in vitro and animal studies (55-58). They are also in agreement with human studies examining endothelium-derived NO activity in the peripheral microvasculature, where reduced bioavailability of NO in patients with hypertension and hypercholesterolemia was demonstrated $(43,44)$. Combined with these observations, our findings suggest that risk factors for atherosclerosis are associated with, and may be a cause of, a generalized abnormality of the vascular endothelium-dependent NO system.

\section{Summary}

In summary, this study demonstrates that constitutive release of NO contributes substantially to resting human coronary vascular tone. ACH-induced coronary vasodilation is largely due to release of NO from the coronary epicardial and microvascular tree. In the presence of risk factors, and despite absence of angiographic evidence for atherosclerosis, there is reduced basal bioavailability of NO from both the coronary epicardial arteries and microvessels. Moreover, patients with risk factors also have reduced stimulated release of NO with $\mathrm{ACH}$.

Our findings imply that patients with risk factors may have increased resting conductance and resistance vessel tone as a result of reduced NO activity, and that because of reduced stimulated release of NO, vasodilation may also be limited during physiologic stresses (27) and therefore contribute to myocardial ischemia in patients exposed to multiple risk factors. Depressed bioavailability of NO may also result in reduced platelet-inhibitory effects of NO (59) and predispose these patients to thrombotic vascular events. Over time, because of the reduced antismooth muscle cell proliferative effects of NO, these individuals may also succumb to more rapid progression of atherosclerosis (60-62).

\section{References}

1. Furchgott, R. F., and J. V. Zawadzki. 1980. The obligatory role of the endothelial cells in the relaxation of arterial smooth muscle by acetylcholine. Nature (Lond.). 288:373-376.

2. Vanhoutte, P. M. 1988. The endothelium. Modulator of vascular smooth muscle tone. N. Engl. J. Med. 319:512-513.

3. Cocks, T. M., and J. A. Angus. 1983. Endothelium-dependent relaxation of coronary arteries by noradrenaline and serotonin. Nature (Lond.). 305:627630.

4. Feletou, M., and P. M. Vanhoutte. 1988. Endothelium-dependent hyperpolarization of canine coronary smooth muscle. Br. J. Pharmacol. 93:515-524.

5. De Mey, J. G., M. Claeys, and P. M. Vanhoutte. 1982. Endotheliumdependent inhibitory effects of acetylcholine, adenosine triphosphate, thrombin and arachidonic acid in the canine femoral artery. J. Pharmacol. Exp. Ther. 222:166-173

6. Holtz, J., U. Forstermann, U. Pohl, M. Giesler, and E. Bassenge. 1984. Flow-dependent, endothelium-mediated dilatation of epicardial coronary arteries in conscious dogs: effects of cyclooxygenase inhibition. J. Cardiovasc. Pharmacol. 6:1161-1169.
7. Rubanyi, G. M., J. C. Romero, and P. M. Vanhoutte. 1986. Flow-induced release of endothelium-derived relaxing factor. Am. J. Physiol. 250(Heart Circ Physiol. 19):1145-1149.

8. Cox, D. A., J. A. Vita, C. B. Treasure, R. D. Fish, R. W. Alexander, O. P. Ganz, and A. P. Selwyn. 1989. Atherosclerosis impairs flow-mediated dilatation of coronary arteries in humans. Circulation. 80:458-465.

9. Drexler, H., A. M. Zeiher, H. Wollschlager, T. Meinertz, H. Just, and T. Bonzel. 1989. Flow-dependent coronary artery dilation in humans. Circulation. 80:466-474.

10. Palmer, R. M., A. G. Ferrige, and S. Moncada. 1987. Nitric oxide release accounts for the biological activity of endothelium-derived relaxing factor. Nature (Lond.). 327:524-526.

11. Ignarro, L. J., R. E. Byrns, G. M. Buga, and K. S. Wood. 1987. Endothelium-derived relaxing factor from pulmonary artery and vein possesses pharmacologic and chemical properties identical to those of nitric oxide radical. Circ. Res. 61:866-879.

12. Palmer, R. M., D. S. Ashton, and S. Moncada. 1988. Vascular endothelial cells synthesize nitric oxide from L-arginine. Nature (Lond.). 333:664-666.

13. Gardiner, S. M., A. M. Compton, T. Bennett, R. M. J. Palmer, and S. Moncada. 1990. Control of regional blood flow by endothelium-derived nitric oxide. Hypertension (Dallas). 15:486-492.

14. Chu, A., D. E. Chambers, C. Lin, W. D. Kuehl, and F. R. Cobb. 1990 Nitric oxide modulates epicardial coronary basal vasomotor tone in awake dogs. Am. J. Physiol. 258:H1250-H1254.

15. Amezcua, J. L., R. M. J. Palmer, B. M. De Souza, and S. Moncada. 1989. Nitric oxide synthesized from L-arginine regulates vascular tone in the coronary circulation of the rabbit. Br. J. Pharmacol. 97:1119-1124.

16. Leipert, B., B. F. Becker, and E. Gerlach. 1992. Different endothelia mechanisms involved in coronary responses to known vasodilators. Am. J. Physiol. 262:H1676-H1683.

17. Tschudi, M., V. Richard, F. R. Buhler, and T. F. Luscher. 1991. Importance of endothelium-derived nitric oxide in porcine coronary resistance arteries. Am. J. Physiol. 260:H13-H20.

18. Parent, R., R. Pare, and M. Lavallee. 1992. Contribution of nitric oxide to dilation of resistance coronary vessels in conscious dogs. Am. J. Physiol. 262:H10-H16.

19. Komarv, T., K. G. Lamping, C. L. Eastham, D. G. Harrison, M. L. Marcos, and K. C. Dellsperger. 1991. Effect of an arginine analogue on acetylcholineinduced coronary microvascular dilatation in dogs. Am. J. Physiol. 261:H2001H2007.

20. Chu, A., D. E. Chambers, C. Lin, W. D. Kuehl, R. M. J. Palmer, S Moncada, and F. R. Cobb. 1991. Effects of inhibition of nitric oxide formation on basal vasomotion and endothelium-dependent responses of the coronary arteries in awake dogs. J. Clin. Invest. 87:1964-1968.

21. Ishizaka, H., K. Okumura, H. Yamabe, T. Tsuchiya, and H. Yasue. 1991 Endothelium-derived nitric oxide as a mediator of acetylcholine-induced coronary vasodilation in dogs. J. Cardiovasc. Pharmacol. 18:665-669.

22. Bassenge, E. 1991. Endothelium-mediated regulation of coronary tone. In Endothelial Mechanisms of Vasomotor Control. H. Drexler, A. M. Zeither, E. Bassenge, and H. Just, editors. Steinkopff Verlag, Darmstadt, Germany. 69-75

23. Ludmer, P. L., A. P. Selwyn, T. L. Shook, R. R. Wayne, G. H. Mudge, R. W. Alexander, and P. Ganz. 1986. Paradoxical vasoconstriction induced by acetylcholine in atherosclerotic coronary arteries. N. Engl. J. Med. 315:10461051 .

24. Zeiher, A. M., H. Drexler, H. Wollschläger, and H. Just. 1991. Modulation of coronary vasomotor tone in humans. Progressive endothelial dysfunction with different early stages of coronary atherosclerosis. Circulation. 83:391-401.

25. Hodgson, J. M., and J. J. Marshall. 1989. Direct vasoconstriction and endothelium-dependent vasodilation. Mechanism of acetylcholine effects on coronary flow and arterial diameter in patients with non-stenotic coronary arteries. Circulation. 79:1043-1051

26. Newman, C. M., A. Maseri, D. R. Hackett, H. M. El-Tamimi, and G. J. Davies. 1990. Responses of angiographically normal and atherosclerotic left anterior descending coronary arteries to acetylcholine. Am. J. Cardiol. 66:1070-1076.

27. Quyyumi, A. A., R. O. Cannon, J. A. Panza, J. G. Diodati, and S. E. Epstein. 1992. Endothelial dysfunction in patients with chest pain and norma coronary arteries. Circulation. 1864-1871.

28. Nabel, E. G., A. P. Selwyn, and P. Ganz. 1990. Paradoxical narrowing of atherosclerotic arteries induced by increases in heart rate. Circulation. 81:850859.

29. Gordon, J. B., P. Ganz, E. G. Nabel, R. D. Fish, J. Zebede, G. H. Mudge, R. W. Alexander, and A. P. Selwyn. 1989. Atherosclerosis influences the vasomotor response of epicardial coronary arteries to exercise. J. Clin. Invest. 83:19461952

30. Yeung, A. C., V. I. Vekshtein, D. S. Krantz, et al. 1991. The effect of atherosclerosis on the vasomotor response of coronary arteries to mental stress. N. Engl. J. Med. 325:1551-1556.

31. Egashira, K., T. Inou, Y. Hirooka, A. Yamada, Y. Urabe, and A. Takeshita. 1993. Evidence of impaired endothelium-dependent coronary vasodilation in pa- 
tients with angina pectoris and normal coronary angiograms. N. Engl. J. Med. 328:1659-1664.

32. Lefroy, D. C., T. Crake, N. G. Uven, G. J. Davies, and A. Maseri. 1993. Effect of inhibition of nitric oxide synthesis on epicardial coronary artery caliber and coronary blood flow in humans. Circulation. 88:43-54.

33. Vita, J. A., C. B. Treasure, E. G. Nabel, J. M. McLenachan, R. D. Fish, A. C. Yeung, V. I. Vekshtein, A. P. Selwyn, and P. Ganz. 1990. Coronary vasomotor response to acetylcholine relates to risk factors for coronary artery disease. Circulation. 81:491-497.

34. Egashira, K., T. Inou, Y. Hirooka, A. Yamade, Y. Maruoka, H. Kai, M. Sugimachi, S. Suzuki, and A. Takeshita. 1993. Impaired coronary blood flow response to acetylcholine in patients with coronary risk factors and proximal atherosclerotic lesions. J. Clin. Invest. 91:29-37.

35. Ofili, E., M. J. Kern, S. Tatineni, U. Deligonul, F. Aguirre, H. Serota, and A. J. Labovitz. 1991. Detection of coronary collateral flow by a Doppler-tipped guide wire during coronary angioplasty. Am. Heart J. 122:221-225.

36. Doucette, J. W., D. Corl, H. M. Payne, A. E. Flynn, M. Goto, M. Nassi, and J. Segal. 1992. Validation of a Doppler guide wire for intravascular measurement of coronary artery flow velocity. Circulation. 85:1899-1911.

37. Rees, D. D., R. M. Palmer, H. F. Hodson, and S. Moncada. 1989. A specific inhibitor of nitric oxide formation from $L$-arginine attenuates endotheliumdependent relaxation. Br. J. Pharmacol. 96:418-424.

38. Glantz, S. A., and B. K. Slinker. 1990. Repeated measures. In Primer of Applied Regression and Analysis of Variance. S. A. Glantz and B. K. Slinker, editors. McGraw-Hill, New York. 381-463.

39. Drexler, H., A. M. Zeiher, T. Meinertz, and H. Just. 1991. Correction of endothelial dysfunction in coronary microcirculation of hypercholesterolemic patients by L-arginine. Lancet. 338:1546-1550.

40. Creager, M. A., X. J. Girerd, S. H. Gallagher, S. Coleman, V. J. Dzau, and J. P. Cooke. 1992. L-arginine improves endothelium-dependent vasodilation in hypercholesterolemic humans. J. Clin. Invest. 90:1248-1253.

41. Chilian, W. M., C. L. Eastham, and M. L. Marcus. 1986. Microvascular distribution of coronary vascular resistance in beating left ventricle. Am. J. Physiol. 251 (Heart Circ. Physiol. 20):H779-H788.

42. Vallance, P., J. Collier, and S. Moncada. 1989. Effects of endotheliumderived nitric oxide on peripheral arteriolar tone in man. Lancet. ii:997-1000.

43. Casino, P. R., C. M. Kilcoyne, A. A. Quyyumi, J. M. Hoeg, and J. A. Panza. 1993. Role of nitric oxide in the endothelium-dependent vasodilation of hypercholesterolemic patients. Circulation. 88:2541-2547.

44. Panza, J. A., P. R. Casino, C. M. Kilcoyne, and A. A. Quyyumi. 1993. Role of endothelium-derived nitric oxide in the abnormal endothelium-dependent vascular relaxation of patients with essential hypertension. Circulation. 87:14681474.

45. Gilligan, D. M., J. A. Panza, C. M. Kilcoyne, J. G. Diodati, P. R. Casino, and A. A. Quyyumi. 1994. The contribution of endothelium-derived nitric oxide to exercise induced vasodilation in man. Circulation. 90:2853-2858.

46. Woodman, O. L., and G. J. Dusting. 1991. N-Nitro L-arginine causes coronary vasoconstriction and inhibits endothelium-dependent vasoconstriction in anaesthetized greyhounds. Br. J. Pharmacol. 103:1407-1410.

47. Flavahan, N. A. 1992. Atherosclerosis or lipoprotein-induced endothelial dysfunction: potential mechanisms underlying reduction in EDRF/nitric oxide activity. Circulation. 85:1927-1938.
48. Ohara, Y., T. E. Peterson, and D. Harrison. 1992. Hypercholesterolemia increases superoxide anion production by the endothelium. Circulation. 86(Suppl. I):I-222. (Abstr.)

49. Minor, R. L., Jr., P. R. Myers, R. Guerra, Jr., J. N. Bates, and D. G. Harrison. 1990. Diet-induced atherosclerosis increases the release of nitrogen oxides from rabbit aorta. J. Clin. Invest. 86:2109-2116.

50. Zeiher, A. M., H. Drexler, H. Wollschläger, and H. Just. 1991. Endothelial dysfunction of the coronary microvasculature is associated with impaired coronary blood flow regulation in patients with early atherosclerosis. Circulation. 84:19841992.

51. Yasue, H., K. Matsuyama, K. Matsuyama, K. Okumura, Y. Morikami, and H. Ogawa. 1990. Responses of angiographically normal human coronary arteries to intracoronary injection of acetylcholine by age and segment. Possible role of early coronary atherosclerosis. Circulation. 81:482-490.

52. Egashira, K., T. Inou, Y. Hirooka, H. Kai, M. Sugimachi, S. Suzuki, T. Kuga, Y. Urabe, and A. Takeshita. 1993. Effects of age on endothelium-dependent vasodilation of resistance coronary arteries by acetylcholine in humans. Circulation. 88:77-81.

53. Zieher, A. M., V. Schächinger, S. H. Hohnloser, B. Saurbier, and H. Just. 1994. Coronary atherosclerotic wall thickening and vascular reactivity in humans: elevated high-density lipoprotein levels ameliorate abnormal vasoconstriction in early atherosclerosis. Circulation. 89:2525-2532.

54. Förstermann, V., A. Mügge, U. Alheid, A. Haverich, and J. C. Frölich. 1988. Selective attenuation of endothelium-mediated vasodilation in atherosclerotic human coronary arteries. Circ. Res. 62:185-190.

55. Yamamoto, H., C. Bossaller, J. Cartwright, Jr., and P. D. Henry. 1988. Videomicroscopic demonstration of defective cholinergic arteriolar vasodilation in atherosclerotic rabbit. J. Clin. Invest. 81:1752-1758.

56. Osborne, J. A., M. J. Siegman, A. W. Sedar, S. U. Mooers, and A. M. Lefer. 1989. Lack of endothelium-dependent relaxation in coronary resistance arteries of atherosclerotic rabbit. Am. J. Physiol. 256:C591-C597.

57. Sellke, F. W., M. L. Armstrong, and D. G. Harrison. 1990. Endotheliumdependent vascular relaxation is abnormal in the coronary microcirculation of atherosclerotic primates. Circulation. 81:1586-1593.

58. Chilian, W. M., K. C. Dellsperger, S. M. Layne, C. L. Easthman, M. A Armstrong, M. L. Marcus, and D. D. Heistad. 1990. Effects of atherosclerosis on the coronary microcirculation. Am. J. Physiol. 258(Heart Circ. Physiol. 27):H529-H539.

59. Yao, S.-K., J. C. Ober, A. Krishnaswami, J. J. Ferguson, V. Anderson, P. Golino, L. M. Buja, and J. T. Willerson. 1992. Endogenous nitric oxide protects against platelet aggregation and cyclic flow variations in stenosed and endothelium-injured arteries. Circulation. 86:1302-1309.

60. Garg, U. C., and A. Hassid. 1989. Nitric oxide-generating vasodilators and 8-bromo-cyclic guanosine monophosphate inhibit mitogenesis and proliferation of cultured rat vascular smooth muscle cells. J. Clin. Invest. 83:1774-1777.

61. Cayatte, A. J., J. J. Palacino, K. Horten, and R. A. Cohen. 1994. Chronic inhibition of nitric oxide production accelerates neointima formation and impairs endothelial function in hypercholesterolemic rabbits. Arterioscler. Thromb. 14:753-759.

62. Naruse, K., M. Shimizu, M. Muramatsu, Y. Toki, Y. Miyazaki, K. Okumura, H. Hashimoto, and T. Ito. 1994. Long-term inhibition of NO synthesis promotes atherosclerosis in the hypercholesterolemic rabbit thoracic aorta. Arterioscler. Thromb. 14:746-752. 